\title{
From Fusion Hierarchy to Excited State TBA
}

\author{
G. Jüttner ${ }^{a, 1}$, A. Klümper ${ }^{a, 2}$ and J. Suzuki ${ }^{b, 3}$ \\ ${ }^{a}$ Universität zu Köln, Institut für Theoretische Physik \\ Zülpicher Str. 77, D-50937, Germany \\ ${ }^{b}$ Institute of Physics, University of Tokyo at Komaba \\ Komaba 3-8-1, Meguro-ku, Tokyo, Japan
}

\begin{abstract}
Functional relations among the fusion hierarchy of quantum transfer matrices give a novel derivation of the TBA equations, namely without string hypothesis. This is demonstrated for two important models of $1 \mathrm{D}$ highly correlated electron systems, the supersymmetric $t-J$ model and the supersymmetric extended Hubbard model. As a consequence, "the excited state TBA" equations, which characterize correlation lengths, are explicitly derived for the $t-J$ model. To the authors' knowledge, this is the first explicit derivation of excited state TBA equations for 1D lattice electron systems.
\end{abstract}

\footnotetext{
${ }^{1}$ E-mail gj@thp.uni-koeln.de

2 E-mail kluemper@thp.uni-koeln.de

3 E-mail suz@hep1.c.u-tokyo.ac.jp
} 


\section{Introduction}

The formulation and application of the string hypothesis has been a story of success as well as a longstanding mystery in the studies of exactly solvable models $[1,2,3,4]$. For finite systems, the deviations from this hypothesis have been discussed $[5,6,7,8]$ and prove essential for a proper description of critical properties $[9,10,11]$. On the other hand, for finite temperatures we expect the hypothesis to give the correct free energy in the thermodynamic limit $[3,4]$.

An alternative approach to thermodynamics which is free of the string hypothesis has been proposed $[14,15,16,17,18,19,20,21,22,23]$. There the essential idea is to deal with $2 \mathrm{D}$ classical counterparts. A novel object, "the quantum transfer matrix" plays a fundamental role. Remarkably, the problem of summing up all states reduces to the evaluation of only the largest eigenvalue of the quantum transfer matrix. Of course, the remaining task is the diagonalization of the quantum transfer matrix. In order to achieve this, a more sophisticated approach has been proposed recently by incorporating the classical integrability structure in two dimensions [20,21,24,25,26,27]. The difficulty still remains in the fact that the resultant system is virtually of finite size with interactions depending on the size.

Locating the Bethe ansatz roots for finite system sizes is another difficult task, which we avoid here by one of the following two alternative strategies. We encode the information of BAE roots for finite size systems by

(1) a finite number of coupled non-linear integral equations for a finite number of unknown functions derived within a direct Bethe ansatz for the diagonalization problem, or

(2) an infinite number of coupled non-linear integral equations for an infinite number of unknown functions obtained within the fusion approach to the diagonalization problem.

Note that both transformations are exact. However, approach (1) has an evident advantage in numerical investigations. Actually several thermodynamical quantities have been explicitly obtained for extensive ranges of temperature and chemical potentials for 1D correlated electron systems within this scheme [25,26,27]. The latter approach (2) would be less important in actual calculations. Nevertheless, it is interesting in its connection with the traditional string approach. The infinite number of unknown functions introduced there, physically corresponds to the fused ("higher spin") transfer matrices. These transfer matrices satisfy algebraic functional relations. For the simplest case, these relations are shown to have a deep mathematical origin, an exact sequence of Yangian modules $[28,29,30,31]$.

In this paper we will show that these functional relations can be transformed into nonlinear integral equations, which are identical to TBA equations. Note that our approach is completely independent of the string hypothesis. It thus gives an independent derivation and further support for the validity of the resultant TBA equations. For the physical applications we need only the eigenvalue of the fundamental ("un-fused") quantum transfer matrix. Thus the introduction of the whole commuting family of transfer matrices seems to be redundant. The point is that the exact relation among them makes the evaluation of the fundamental one possible. This fact was first realized in [20] for the RSOS chains. In this paper, we will apply this scheme to two 1D highly correlated electron models to 
demonstrate that the relation of the fusion analysis of the quantum transfer matrix and the TBA is not accidental but universal.

The novel formalism, utilizing the functional relations, not only reproduces the results obtained from the string hypothesis, but goes well beyond. TBA equations usually deal with the equilibrium free energy, i.e. we can only deal with the "largest eigenvalue sector" in the sense of the quantum transfer matrix. The functional relations are, on the other hand, valid for arbitrary eigenvalue sectors. The latter, therefore offers a wider possibility in calculating various physical quantities. Actually, we need to evaluate the subleading eigenvalues for obtaining correlation lengths. Recently, some attempts have been made in the context of deformed conformal field theories, to modify TBA equations so as to deal with excited states $[33,34,32,20,36,37,38]$. We will show how the quantum transfer matrix formalism naturally leads to excited state TBA equations. To our knowledge, this is the first explicit derivation of the excited state TBA equations for 1D lattice electron systems.

This paper is organized as follows. In the next section, we will give a brief review of the quantum transfer matrix approach to one-dimensional quantum systems at finite temperatures. In section 3, the functional relations among the relevant transfer matrices are given. The corresponding relations for the ordinary row-to-row transfer matrices were obtained recently [39]. Here, we will summarize the necessary modifications for the quantum transfer matrices. Section 4 and 5 are devoted to the thermodynamics of the integrable $t-J$ model and the supersymmetric extended Hubbard model, respectively. We will show explicitly how the functional relations of section 3 can be transformed in the case of the largest eigenvalue into the TBA equations of $[40,41]$. To this end, we adopt assumptions about the analyticity of auxiliary function, which are checked numerically for the case of finite Trotter numbers. In section 6, we will discuss "excitations at finite temperatures", i.e. the correlation lengths of static two point functions. Assisted by numerical investigations, the excited state TBA equations are easily derived there. Section 7 is devoted to the summary and discussion. 


\section{The quantum transfer matrix formalism}

In the following we will mainly focus on two specific models of $1 \mathrm{D}$ highly correlated electron systems: the supersymmetric $t-J$ model $[43,40,44,45]$ and the supersymmetric extended Hubbard (SEH) model [42]. They describe spin-1/2 electron systems on the lattice. The prototype of such systems is the celebrated Hubbard model. The supersymmetric $t-J$ model may be viewed as the large Coulomb repulsion limit of a generalized Hubbard model. The SEH model on the other hand, generalizes the Hubbard model by additional bondcharge interactions etc. It shares the same interesting physical symmetry with the standard Hubbard model resulting in the eta pairing $[46,47]$. Mathematically the supersymmetric $t-J$ model and SEH model are in the category of models based on Lie superalgebras $g l(r \mid s)$ with $r=2, s=1$ for the $t-J$ model and $r=s=2$ for the SEH model. As remarked in $[50,51,24]$, their classical counterparts are special cases of the Perk-Schulz [48,49] model with rational vertex weights $\mathcal{R}_{\alpha \beta}^{\mu \nu}$

$$
\begin{aligned}
& \mathcal{R}_{\alpha \alpha}^{\alpha \alpha}(v)=1+\epsilon_{\alpha} v \\
& \mathcal{R}_{\alpha \alpha}^{\mu \mu}(v)=\epsilon_{\alpha} \epsilon_{\mu} v \\
& \mathcal{R}_{\mu \alpha}^{\alpha \mu}(v)=1
\end{aligned}
$$

which satisfy the Yang-Baxter equation. As a consequence the row-to-row transfer matrix $\mathcal{T}(v)$ constitutes a commuting family with respect to the spectral variable $v . \epsilon_{\alpha}= \pm 1$ are discrete parameters determining the 'grading' of the system. The Hamiltonian of the associated quantum system is given by the logarithmic derivative

$$
\mathcal{H}=\left.\frac{d}{d v} \ln \mathcal{T}(v)\right|_{v=0} .
$$

The supersymmetric $t-J$ Hamiltonian is obtained for $\left(\epsilon_{1}, \epsilon_{2}, \epsilon_{3}\right)=(+,-,+)$. Likewise the grading $\left(\epsilon_{1}, \epsilon_{2}, \epsilon_{3}, \epsilon_{4}\right)=(+,-,-,+)$ leads to the SEH model.

Next we introduce Boltzmann weights $\overline{\mathcal{R}}$ and $\widetilde{\mathcal{R}}$ related to $\mathcal{R}$ by anti-clockwise and clockwise $90^{\circ}$ rotations

$$
\overline{\mathcal{R}}_{\alpha \beta}^{\mu \nu}(v)=\mathcal{R}_{\nu \mu}^{\alpha \beta}(v), \quad \widetilde{\mathcal{R}}_{\alpha \beta}^{\mu \nu}(v)=\mathcal{R}_{\mu \nu}^{\beta \alpha}(-v) .
$$

According to (2.1), (2.2) we find

$$
\mathcal{T}(v)=\mathcal{T}_{R} \mathrm{e}^{v \mathcal{H}+\mathcal{O}\left(v^{2}\right)}, \quad \overline{\mathcal{T}}(v)=\mathcal{T}_{L} \mathrm{e}^{v \mathcal{H}+\mathcal{O}\left(v^{2}\right)},
$$

where $\mathcal{T}_{R, L}$ are right and left-shift operators. Therefore the partition function of the quantum system is given by $[17,20,22]$

$$
Z=\lim _{N \rightarrow \infty} \operatorname{Tr}[\mathcal{T}(u) \overline{\mathcal{T}}(u)]^{N / 2}, \quad u=-\beta / N .
$$

The right-hand side of this equation may be viewed as the partition function of a staggered Perk-Schulz model consisting of alternating rows of $\mathcal{R}(u)$ and $\overline{\mathcal{R}}(u)$ operators. For the 
following analytic calculations the column-to-column transfer matrix $\mathcal{T}^{Q T M}$ (quantum transfer matrix) is best adapted. For quite general systems it can be shown that $\mathcal{T}^{Q T M}$ possesses a gap between the largest and next-largest eigenvalues $\Lambda_{\text {max }}$ and $\Lambda_{\text {next }}$ persisting in the limit $N \rightarrow \infty$. Therefore, the problem of computing the free energy $f$ per site of the quantum system at finite temperature is reduced to just the evaluation of the largest eigenvalue $\Lambda_{\max }$

$$
f=-k_{B} T \ln \Lambda_{\max }, \quad(N \rightarrow \infty) .
$$

If also the gap can be calculated (as shown below) the correlation length at finite temperature can be derived

$$
\xi=\left(\ln \frac{\Lambda_{\max }}{\Lambda_{\text {next }}}\right)^{-1}, \quad(N \rightarrow \infty)
$$

The quantum transfer matrix is given by an alternating product of $\mathcal{R}(u)$ and $\widetilde{\mathcal{R}}(-u)$ operators. The important observation $[20,52]$ is that $\widetilde{\mathcal{R}}(v)$ and $\mathcal{R}(v)$ share the same intertwiner as can be proven most easily in a graphical way, see Fig.1. Therefore

$$
\mathcal{T}^{\mathrm{QTM}}(u, v)=\prod_{i=1}^{N / 2 \otimes} \mathcal{R}(v+u) \otimes \widetilde{\mathcal{R}}(v-u)
$$

represents a family of commuting matrices comprising the 'physical' QTM at $v=0$. This intertwining property implies that the Yangian algebra, in the realization $R L L=L L R$, is identical for both monodromy matrices. Consequently, we should have the same functional relations among the transfer matrices in spite of apparent differences between the explicit eigenvalues. Of course this argument needs further elaboration. However, we leave it as an interesting future problem and assume the validity of the above argument in the following. 


\section{The fusion hierarchy}

First, we like to sketch the strategy for the diagonalization of the quantum transfer matrix for the Perk-Schulz model spectral parameters $u, i v$ (where we have introduced the factor $i$ for later convenience). In the following analysis we will be dealing with transfer matrices formulated for fused Boltzmann weights. These matrices are denoted by $T_{m}^{(a)}(u, v)$ where $T_{1}^{(1)}(u, v)=\mathcal{T}^{\mathrm{QTM}}(u, i v)$. Of course $T_{1}^{(1)}(u, 0)$ is the only quantity we are interested in. For calculating the eigenvalues of $T_{1}^{(1)}(u, 0)$ it is essential to deal with all $T_{m}^{(a)}(u, v)$ as our method employs functional equations dealing with the dependence on $v$ and all other matrices for any $a, m[20]$.

In order to present our results for the eigenvalue of the quantum transfer matrix we adopt a compact notation using the "Yangian analogue of Young tableaux" [53,54,55]. First consider the simplest case: the quantum transfer matrix with $r+s$ dimensional degrees of freedom on both vertical and horizontal edges. Let us introduce boxes with a letter $\in\{1, \cdots, r+s\}$. Each box corresponds to an expression as follows.

$$
\mathrm{a}_{v}=f_{a}(u+i v) g_{a}(u-i v) \epsilon_{a}^{N_{a-1}+N_{a}} \frac{Q_{a-1}\left(v-i \epsilon_{a}\right)}{Q_{a-1}(v)} \frac{Q_{a}\left(v+i \epsilon_{a}\right)}{Q_{a}(v)},
$$

where

$$
\begin{aligned}
f_{a}(x) & = \begin{cases}\left(1+\epsilon_{1} x\right)^{N / 2}, & \text { for } a=1, \\
x^{N / 2}, & \text { otherwise },\end{cases} \\
g_{a}(x) & = \begin{cases}\left(1+\epsilon_{r+s} x\right)^{N / 2}, & \text { for } a=r+s, \\
x^{N / 2}, & \text { otherwise },\end{cases} \\
Q_{a}(x) & = \begin{cases}\prod_{j=1}^{N_{a}}\left(x-x_{j}^{(a)}\right), & \text { for } a=1, \cdots, r+s-1, \\
1, & \text { for } a=0, r+s .\end{cases}
\end{aligned}
$$

Note that a box carries the spectral parameter dependence. In the last equation, $x_{j}^{(a)}(j=$ $1, \cdots, N_{a}$ ) denotes the Bethe ansatz roots with "color" $a$. (We adopt the convention $\left.N_{0}=N_{r+s}=0\right)$. As derived by an algebraic Bethe ansatz the eigenvalue $\Lambda_{1}^{(1)}(u, v)$ of the quantum transfer matrix is of the form

$$
\Lambda_{1}^{(1)}(u, v)=\sum_{a=1}^{r+s} \mathrm{a}_{v} .
$$

The Bethe ansatz equations are obtained by the pole free condition for the eigenvalue. The BAEs are therefore given by

$$
\begin{aligned}
& \operatorname{Res}_{v=x_{j}^{(a)}}\left(\mathrm{a}_{v}+\mathrm{a}+1_{v}\right)=0, \\
& -\epsilon_{a}^{N_{a-1}+N_{a}} \epsilon_{a+1}^{N_{a}+N_{a+1}} \frac{f_{a}\left(u+i x_{j}^{(a)}\right) g_{a}\left(u-i x_{j}^{(a)}\right)}{f_{a+1}\left(u+i x_{j}^{(a)}\right) g_{a+1}\left(u-i x_{j}^{(a)}\right)}= \\
& \frac{Q_{a-1}\left(x_{j}^{(a)}\right) Q_{a}\left(x_{j}^{(a)}-i \epsilon_{a+1}\right) Q_{a+1}\left(x_{j}^{(a)}+i \epsilon_{a+1}\right)}{Q_{a-1}\left(x_{j}^{(a)}-i \epsilon_{a}\right) Q_{a}\left(x_{j}^{(a)}+i \epsilon_{a}\right) Q_{a+1}\left(x_{j}^{(a)}\right)}
\end{aligned}
$$


Starting from $\Lambda_{1}^{(1)}$ as in [54], one can generate a set of analytic functions under the BAE. The resultant functions are expected to be eigenvalues of fusion transfer matrices. For the simplest case like $s l_{2}$ spin chains, we can explicitly prove this $[56,57]$. We do not attempt to prove this observation for the general case as our analytic treatment below does not depend on it. In the case of row-to-row transfer matrices, the "Bethe-strap procedure" has been executed for $s l(r \mid s)$ models [39]. The eigenvalues of fusion transfer matrices are parameterized by a similar set of tableaux under certain combinatorial rules. A set of functional relations among them is also found. As remarked above, the same set of functional relations holds for the quantum transfer matrices as well as the combinatorial rules for the tableaux giving the analytic eigenvalues for the fusion transfer matrices. If one replaces boxes for the row-to-row case by the quantum ones in eq (3.1), then the explicit eigenvalues of "fused transfer matrices" are obtained. Let us further explain the rules in the present context. Consider an $a \times m$ rectangular tableaux. We fix the "coordinate" so that the upper-leftmost box is in the position $(1,1)$, the one box to the right is in $(1,2)$ and the one box lower is in $(2,1)$. We associate the spectral parameter $v+i(m-a) / 2+i(j-k)$ with the box in $(j, k)$. Denote the letter in the $(j, k)$ box by $\ell_{j, k}$. We identify such tableaux with the expression

$$
\prod_{j=1}^{a} \prod_{k=1}^{m} \ell_{j, k}{ }_{v+i(m-a) / 2+i(j-k)} .
$$

To describe the combinatorial rules we introduce the order among letters, $1 \prec 2 \prec \cdots \prec$ $r+s$. These letters are further classified into two subsets $J_{+}$and $J_{-}$. Then the admissibility conditions are [39]

(1) $\ell_{j-1, k} \prec \ell_{j, k}$ if $\ell_{j, k} \in J_{+}$

(2) $\ell_{j-1, k} \preceq \ell_{j, k}$ if $\ell_{j, k} \in J_{-}$

(3) $\ell_{j, k-1} \preceq \ell_{j, k} \quad$ if $\ell_{j, k} \in J_{+}$

(4) $\ell_{j, k-1} \prec \ell_{j, k}$ if $\ell_{j, k} \in J_{-}$

Define $\Lambda_{m}^{(a)}(u, v)$ by

$$
\Lambda_{m}^{(a)}(u, v)=\sum_{\left\{\ell_{j, k}\right\} \text { under rules }(1)-(4)} \prod_{j=1}^{a} \prod_{k=1}^{m}{\sum \ell_{j, k}}_{v+i(m-a) / 2+i(j-k)} .
$$

$\Lambda_{m}^{(a)}(u, v)$ is expected to be the eigenvalue of the quantum transfer matrix whose trace has been taken over the Yangian module $W_{m}^{(a)}$. We have one further rule [58]:

$$
\Lambda_{m}^{(a)}(u, v)=0 \quad \text { if } a>r \text { and } m>s .
$$

The functional relations read

$$
\Lambda_{m}^{(a)}\left(u, v-\frac{i}{2}\right) \Lambda_{m}^{(a)}\left(u, v+\frac{i}{2}\right)=\Lambda_{m}^{(a+1)}(u, v) \Lambda_{m}^{(a-1)}(u, v)+\Lambda_{m-1}^{(a)}(u, v) \Lambda_{m+1}^{(a)}(u, v)
$$

with the convention $\Lambda_{0}^{(a)}=\Lambda_{m}^{(0)}=1$. These relations can be proved using a quantum analogue of the Jacobi-Trudi formula and Plücker's relation $[55,39]$. Recently some attempts 
have been made in the study of finite-size corrections in $t-J$ like models employing similar functional relations [59]. There the row-to-row transfer matrix was considered and a subset of fusion functions was used.

In the next two sections, we will transform the complete set of functional equations into integral form which is useful for further analytic treatment. We will show explicitly how the simple functional relations above are nothing but TBA equations for the quantum chains related to the Perk-Schulz model. We will focus on two specific examples of interests, the integrable $t-J$ model and the SEH model.

\section{Fusion based derivation of TBA for the supersymmetric $t-J$ model}

For the $t-J$ model, we choose a convenient grading $\epsilon_{1}=\epsilon_{3}=1, \epsilon_{2}=-1$. Then $J_{+}=$ $\{1,3\}, J_{-}=\{2\}$. For example, we have the following five tables for $\Lambda_{2}^{(1)}$

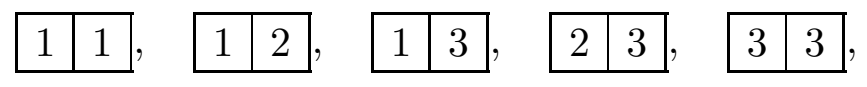

and four tables for $\Lambda_{1}^{(2)}$,

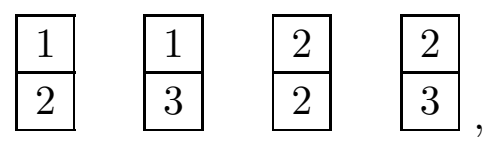

and so on. From the analytic point of view, it is better to divide the $\Lambda_{m}^{(a)}(u, v)$ as defined in (3.5) by a common factor, such that the resultant expression is a polynomial of degree $N$ in $v$ irrespective of the values of $a$ and $m$. For that purpose we prepare the normalization functions,

$$
\begin{aligned}
h_{m}^{(1)}(x)= & \prod_{j=1}^{m-1} \phi_{-}\left(x+\left(j-\frac{(m+1)}{2}\right) i\right) \phi_{+}\left(x-\left(j-\frac{(m+1)}{2}\right) i\right), \\
n_{m}^{(a)}(x)= & \prod_{j=1}^{a} h_{m}^{(1)}\left(x+\left(j-\frac{a+1}{2}\right) i\right) \times \\
& \prod_{j=1}^{a-1} \phi_{-}\left(x+\frac{(m+a-2 j)}{2} i\right) \phi_{+}\left(x-\frac{(m+a-2 j)}{2} i\right),
\end{aligned}
$$

where $\phi_{ \pm}(v)=(v \pm i u)^{N / 2}$. Then the normalized functions $\widetilde{\Lambda_{m}^{(a)}}$ are given by

$$
\begin{aligned}
\Lambda_{m}^{(a)}(u, x) & =n_{m}^{(a)}(x) \widetilde{\Lambda_{m}^{(a)}}(x), \\
\widetilde{\Lambda_{m}^{(0)}}(x) & =\phi_{+}\left(x-\frac{m}{2} i\right) \phi_{-}\left(x+\frac{m}{2} i\right), \\
\widetilde{\Lambda_{0}^{(a)}}(x) & =\phi_{+}\left(x+\frac{a}{2} i\right) \phi_{-}\left(x-\frac{a}{2} i\right) .
\end{aligned}
$$

Hereafter we drop $u$ from $\widetilde{\Lambda_{m}^{(a)}}$ for simplicity. 
Now the functional relations read,

$$
\begin{aligned}
& \widetilde{\Lambda_{m}^{(1)}}(x-i / 2) \widetilde{\Lambda_{m}^{(1)}}(x+i / 2)=\widetilde{\Lambda_{m+1}^{(1)}}(x) \widetilde{\Lambda_{m-1}^{(1)}}(x)+\widetilde{\Lambda_{m}^{(2)}}(x) \widetilde{\Lambda_{m}^{(0)}}(x), \quad m=1, \cdots, \infty, \widetilde{\Lambda^{(1)}}(x) \widetilde{\Lambda_{1}^{(3)}}(x), \\
& \widetilde{\Lambda_{1}^{(2)}}(x-i / 2) \widetilde{\Lambda_{1}^{(2)}}(x+i / 2)=\widetilde{\Lambda_{2}^{(2)}}(x) \widetilde{\Lambda_{0}^{(2)}}(x)+\widetilde{\Lambda_{1}^{(1)}}\left(\widetilde{\Lambda_{m}^{(2)}}(x+i / 2)=\widetilde{\Lambda_{m+1}^{(2)}}(x) \widetilde{\Lambda_{m-1}^{(2)}}(x), \quad m=2, \cdots, \infty, \widetilde{\Lambda_{m}^{(2)}}(x-i / 2)\right. \\
& \widetilde{\Lambda^{(a)}}(x-i / 2) \widetilde{\Lambda_{1}^{(a)}}(x+i / 2)=\widetilde{\Lambda_{1}^{(a+1)}}(x) \widetilde{\Lambda_{1}^{(a-1)}}(x), \quad a=3, \cdots, \infty .
\end{aligned}
$$

In the above, not all $\widetilde{\Lambda_{m}^{(a)}}(x)$ are independent; we have the identity $\widetilde{\Lambda_{1}^{(a+1)}}(x)=\widetilde{\Lambda_{a}^{(2)}}(x)$, which can be easily proved by explicit forms. Now we apply transformations generalizing the ones in $[20,57,31]$

$$
\begin{aligned}
Y_{m}^{(1)}(x) & =\frac{\widetilde{\Lambda_{m+1}^{(1)}}(x) \widetilde{\Lambda_{m-1}^{(1)}}(x)}{\widetilde{\Lambda_{m}^{(2)}}(x) \widetilde{\Lambda_{m}^{(0)}}(x)}, \quad \text { for } m \geq 1, \\
Y_{1}^{(a)}(x) & =\frac{\widetilde{\Lambda_{0}^{(a)}}(x)}{\widetilde{\Lambda_{1}^{(a-1)}}(x)}, \quad \text { for } a \geq 2 .
\end{aligned}
$$

We call the functional relations in terms of the functions $Y_{m}^{(a)}(x)$ simply the "Y-system" $[12,35,60]$. The explicit $\mathrm{Y}$-system is now given by

$$
\begin{aligned}
Y_{1}^{(1)}\left(x-\frac{i}{2}\right) Y_{1}^{(1)}\left(x+\frac{i}{2}\right) & =\frac{\left(1+Y_{2}^{(1)}(x)\right)}{\left(1+\left(Y_{1}^{(2)}(x)\right)^{-1}\right)}, \\
Y_{m}^{(1)}\left(x-\frac{i}{2}\right) Y_{m}^{(1)}\left(x+\frac{i}{2}\right) & =\left(1+Y_{m+1}^{(1)}(x)\right)\left(1+Y_{m-1}^{(1)}(x)\right), \quad \text { for } m \geq 2, \\
Y_{1}^{(2)}\left(x-\frac{i}{2}\right) Y_{1}^{(2)}\left(x+\frac{i}{2}\right) & =\frac{\phi_{+}(x+i / 2) \phi_{-}(x-i / 2)}{\phi_{-}(x+i / 2) \phi_{+}(x-i / 2)} \frac{Y_{1}^{(3)}(x)}{\left(1+Y_{1}^{(1)}(x)\right)}, \\
Y_{1}^{(3)}\left(x-\frac{i}{2}\right) Y_{1}^{(3)}\left(x+\frac{i}{2}\right) & =\frac{Y_{1}^{(4)}(x)}{\left(1+\left(Y_{1}^{(2)}(x)\right)-1\right)}, \\
Y_{1}^{(a)}\left(x-\frac{i}{2}\right) Y_{1}^{(a)}\left(x+\frac{i}{2}\right) & =Y_{1}^{(a+1)}(x) Y_{1}^{(a-1)}(x), \quad \text { for } a \geq 4 .
\end{aligned}
$$

At this stage we note some numerical results concerning the analytic properties of $\widetilde{\Lambda_{m}^{(1)}}(x)$. They are analytic due to the BAE, and have $N$ zeros. Through finite $N$ studies, we find their characteristic behavior. For the eigenstate giving the largest eigenvalue for $\widetilde{\Lambda_{1}^{(1)}}(x)$, the zeros are located on curved lines with imaginary parts close to $\pm(m+1) / 2$. Therefore all $Y_{m}^{(a)}(x)$ except for $a=m=1$ are Analytic Nonzero and have Constant asymptotics(ANZC) in the strip $\Im x \in\{-1 / 2,1 / 2\}$. Similarly we have numerically checked that $1+Y_{m}^{(a)}(x)$ has 
the ANZC property in this strip. For the case of $\left(1+Y_{2}^{(1)}\right)$ see Fig. 2. To cancel zeros and holes for $Y_{1}^{(1)}(x)$, we define

$$
\widetilde{Y_{m}^{(a)}(x)}= \begin{cases}\left(\tanh \frac{\pi}{2}\left(x+i\left(\frac{1}{2}+u\right)\right) \tanh \frac{\pi}{2}\left(x-i\left(\frac{1}{2}+u\right)\right)\right)^{-N / 2} Y_{m}^{(a)}(x), & \text { for } \mathrm{a}=\mathrm{m}=1 \\ Y_{m}^{(a)}(x), & \text { otherwise }\end{cases}
$$

Now the left hand sides of eqs. (4.5) are invariant if we replace $Y_{m}^{(a)}(x)$ by $\widetilde{Y_{m}^{(a)}}(x)$. After this replacement, both sides of eqs. (4.5) are ANZC functions in a narrow strip including the real axis. Therefore the Fourier transformation of logarithms of both sides can be simplified due to Cauchy's theorem. This results in a set of linear equations in Fourier space which can be solved easily. The reverse Fourier transform leads to the following infinitely many coupled non-linear integral equations.

$$
\begin{aligned}
& \log Y_{1}^{(1)}(x)=e_{1}(u, x)+G_{0} * \log \left(1+Y_{2}^{(1)}\right)(x)-G_{0} * \log \left(1+\frac{1}{Y_{1}^{(2)}}\right)(x), \\
& \log Y_{m}^{(1)}(x)=G_{0} * \log \left(1+Y_{m-1}^{(1)}\right)(x)+G_{0} * \log \left(1+Y_{m+1}^{(1)}\right)(x), \quad \text { for } m \geq 2, \\
& \log Y_{1}^{(2)}(x)=e_{2}(u, x)+G_{0} * \log Y_{1}^{(3)}(x)-G_{0} * \log \left(1+Y_{1}^{(1)}\right)(x), \\
& \log Y_{1}^{(3)}(x)=G_{0} * \log Y_{1}^{(4)}(x)-G_{0} * \log \left(1+\frac{1}{Y_{1}^{(2)}}\right), \\
& \log Y_{1}^{(a)}(x)=G_{0} * \log Y_{1}^{(a-1)}(x)+G_{0} * \log Y_{1}^{(a+1)}(x), \quad \text { for } a \geq 4 .
\end{aligned}
$$

Here $A * B(x)$ means convolution $A * B=\int A(x-y) B(y) d y$, and

$$
\begin{aligned}
e_{1}(u, x) & =\log \left(\tanh \frac{\pi}{2}\left(x+i\left(\frac{1}{2}+u\right)\right) \tanh \frac{\pi}{2}\left(x-i\left(\frac{1}{2}+u\right)\right)\right)^{N / 2}, \\
e_{2}(u, x) & =N \int \frac{e^{-|k| / 2-i k x} \sinh k u}{2 k \cosh k / 2} \mathrm{dk}, \\
G_{0}(x) & =\frac{1}{2 \pi} \int \frac{e^{-i k x}}{2 \cosh k / 2}=\frac{1}{2 \cosh \pi x} .
\end{aligned}
$$

Note that $\log Y_{1}^{(3)}(x)$ can be solved in term of $\log \left(1+\frac{1}{Y_{1}^{(2)}}\right)$ from the last two relations in the above. Resubstitution of the result into the third relation above leads to

$$
\log Y_{1}^{(2)}(x)=e_{2}(u, x)-G_{1} * \log \left(1+\frac{1}{Y_{1}^{(2)}}\right)(x)-G_{0} * \log \left(1+Y_{1}^{(1)}\right)(x)
$$

where $G_{1}(x)=\frac{1}{2 \pi} \int d k \frac{e^{-i k x-|k| / 2}}{2 \cosh k / 2}$.

Therefore we have the resultant Y-system including $Y_{m}^{(1)},(m=1, \cdots, \infty)$ and $Y_{1}^{(2)}$. The quantity of our interest, the free energy, is given in terms of $Y_{m}^{(a)}$ as,

$$
-\beta f=\lim _{N \rightarrow \infty} \log \Lambda_{1}^{(1)}\left(u=-\frac{\beta}{N}, 0\right)=\lim _{N \rightarrow \infty} \log \phi_{+}(i) \phi_{-}(-i)-\log Y_{1}^{(2)}\left(u=-\frac{\beta}{N}, 0\right)
$$


Now the limit $N \rightarrow \infty$ is taken easily by analytic means. To write down the final result, we make slight changes of notations

$$
\begin{aligned}
& Y_{1}^{(1)}(x)=\exp (-\epsilon(x) / T) \\
& Y_{m}^{(1)}(x)=\exp \left(\phi_{m-1}(x) / T\right), \quad m \geq 2 \\
& Y_{1}^{(2)}(x)=\exp (-\psi(x) / T)
\end{aligned}
$$

Then we have

$$
\begin{aligned}
\epsilon & =2 \pi G_{0}+T G_{0} * \log \frac{\left(1+e^{\psi / T}\right)}{\left(1+e^{\phi_{1} / T}\right)}, \\
\psi & =2 \pi G_{1}+T G_{0} * \log \left(1+e^{-\epsilon / T}\right)+T G_{1} * \log \left(1+e^{\psi / T}\right), \\
\phi_{1} & =T G_{0} * \log \left[\left(1+e^{-\epsilon / T}\right)\left(1+e^{\phi_{2} / T}\right)\right], \\
\phi_{m} & =T G_{0} * \log \left[\left(1+e^{\phi_{m-1} / T}\right)\left(1+e^{\phi_{m+1} / T}\right)\right], \\
f & =1-\psi(0) .
\end{aligned}
$$

They are nothing but the TBA equations obtained in [40] for $x_{s}=1, A^{\prime}=0$. The shift by unity in the free energy is due to a trivial shift in the definition of the Hamiltonian. Thus we have succeeded in deriving these equations independent of the string hypothesis. In the next section, we further apply the same strategy to another model in 1D correlated electron systems to convince ourselves of the universality of the result.

\section{TBA for the SEH model}

For this model, we choose the grading $\left\{\epsilon_{1}, \epsilon_{2}, \epsilon_{3}, \epsilon_{4}\right\}=\{+,-,-,+\}$. Then $J_{+}=\{1,4\}$, $J_{-}=\{2,3\}$. Due to these choices, we have non-vanishing $\Lambda^{\prime}$ 's: $\Lambda_{m}^{(1)}, \Lambda_{m}^{(2)}, \Lambda_{1}^{(a)}, \Lambda_{2}^{(a)},(m=$ $0, \cdots, \infty, a=0, \cdots, \infty)$. While the derivations of TBA based on the string hypothesis for the $t-J$ and the SEH seem to be different, we find them quite similar by adopting the present strategy. We thus sketch the relevant definitions and relations for the SEH model, without going into details. As for the $t-J$ model, we re-normalize $\Lambda_{m}^{(a)}(u, v)$ by the same $n_{m}^{(a)}(u, v)$ in (4.1.b). The renormalized functional relations are given by

$$
\begin{aligned}
& \widetilde{\Lambda_{m}^{(1)}}(x-i / 2) \widetilde{\Lambda_{m}^{(1)}}(x+i / 2)=\widetilde{\Lambda_{m+1}^{(1)}}(x) \widetilde{\Lambda_{m-1}^{(1)}}(x)+\widetilde{\Lambda_{m}^{(2)}}(x) \widetilde{\Lambda_{m}^{(0)}}(x), m=1, \cdots, \infty \\
& \widetilde{\Lambda_{1}^{(a)}}(x-i / 2) \widetilde{\Lambda_{1}^{(a)}}(x+i / 2)=\widetilde{\Lambda_{2}^{(a)}}(x) \widetilde{\Lambda_{0}^{(a)}}(x)+\widetilde{\Lambda_{1}^{(a-1)}}(x) \widetilde{\Lambda_{1}^{(a+1)}}(x), \quad a=2, \cdots \\
& \widetilde{\Lambda_{2}^{(2)}}(x-i / 2) \widetilde{\Lambda_{2}^{(2)}}(x+i / 2)=\widetilde{\Lambda_{3}^{(2)}}(x) \widetilde{\Lambda_{1}^{(2)}}(x)+\widetilde{\Lambda_{2}^{(1)}}(x) \widetilde{\Lambda_{2}^{(3)}}(x), \\
& \widetilde{\Lambda_{m}^{(2)}}(x-i / 2) \widetilde{\Lambda_{m}^{(2)}}(x+i / 2)=\widetilde{\Lambda_{m+1}^{(2)}}(x) \widetilde{\Lambda_{m-1}^{(2)}}(x), \quad m=3, \cdots, \infty, \\
& \widetilde{\Lambda_{2}^{(a)}}(x-i / 2) \widetilde{\Lambda_{2}^{(a)}}(x+i / 2)=\widetilde{\Lambda_{2}^{(a+1)}}(x) \widetilde{\Lambda_{2}^{(a-1)}}(x), \quad a=3, \cdots, \infty \text {. }
\end{aligned}
$$

In addition we have the equalities,

$$
\widetilde{\Lambda_{m}^{(2)}}(x)=\widetilde{\Lambda_{2}^{(m)}}(x), \quad m \geq 2 .
$$


We introduce the $Y$ functions as follows

$$
\begin{aligned}
& Y_{m}^{(1)}(x)=\frac{\widetilde{\Lambda_{m+1}^{(1)}}(x) \widetilde{\Lambda_{m-1}^{(1)}}(x)}{\widetilde{\Lambda_{m}^{(2)}}(x) \widetilde{\Lambda_{m}^{(0)}}(x)}, \quad \text { for } m \geq 1, \\
& Y_{m}^{(2)}(x)=\frac{\widetilde{\Lambda_{m+1}^{(2)}}(x) \widetilde{\Lambda_{m-1}^{(2)}}(x)}{\widetilde{\Lambda_{m}^{(3)}}(x) \widetilde{\Lambda_{m}^{(1)}}(x)}, \quad \text { for } m=1,2, \\
& Y_{1}^{(a)}(x)=\frac{\widetilde{\Lambda_{2}^{(a)}}(x) \widetilde{\Lambda_{0}^{(a)}}(x)}{\widetilde{\Lambda_{1}^{(a+1)}}(x) \widetilde{\Lambda_{1}^{(a-1)}}(x)}, \quad \text { for } a \geq 3 .
\end{aligned}
$$

Then the following Y-system holds,

$$
\begin{aligned}
Y_{1}^{(1)}\left(x-\frac{i}{2}\right) Y_{1}^{(1)}\left(x+\frac{i}{2}\right) & =\frac{\left(1+Y_{2}^{(1)}(x)\right)}{\left(1+\left(Y_{1}^{(2)}(x)\right)^{-1}\right)}, \\
Y_{2}^{(1)}\left(x-\frac{i}{2}\right) Y_{2}^{(1)}\left(x+\frac{i}{2}\right) & =\frac{\left(1+Y_{3}^{(1)}(x)\right)\left(1+Y_{1}^{(1)}(x)\right)}{\left(1+\left(Y_{2}^{(2)}(x)\right)^{-1}\right)}, \\
Y_{m}^{(1)}\left(x-\frac{i}{2}\right) Y_{m}^{(1)}\left(x+\frac{i}{2}\right) & =\left(1+Y_{m+1}^{(1)}(x)\right)\left(1+Y_{m-1}^{(1)}(x)\right), \quad \text { for } m \geq 3, \\
Y_{1}^{(2)}\left(x-\frac{i}{2}\right) Y_{1}^{(2)}\left(x+\frac{i}{2}\right) & =\frac{\left(1+Y_{2}^{(2)}(x)\right)}{\left(1+\left(Y_{1}^{(3)}(x)\right)^{-1}\right)\left(1+\left(Y_{1}^{(1)}(x)\right)^{-1}\right)}, \\
Y_{1}^{(a)}\left(x-\frac{i}{2}\right) Y_{1}^{(a)}\left(x+\frac{i}{2}\right) & =Y_{1}^{(a+1)}(x) Y_{1}^{(a-1)}(x), \quad \text { for } a \geq 3, \\
Y_{2}^{(2)}\left(x-\frac{i}{2}\right) Y_{2}^{(2)}\left(x+\frac{i}{2}\right) & =\frac{\phi_{+}(x+i) \phi_{-}(x-i)}{\phi_{-}(x+i) \phi_{+}(x-i)} \frac{\left(1+\left(Y_{1}^{(2)}(x)\right)^{-1}\right)}{\left(1+Y_{2}^{(1)}(x)\right)} .
\end{aligned}
$$

Numerically, both sides of the above equations have the ANZC property in a narrow strip near the real axis, except for the lhs of the first equation. As for the $t-J$ model, we cancel zeros and singularities by multiplying the scalar as in (4.6). Then the Y-system is equivalent to the following set of non-linear integral equations

$$
\begin{aligned}
& \log Y_{1}^{(1)}(x)=e_{1}(u, x)+G_{0} * \log \left(1+Y_{2}^{(1)}\right)(x)-G_{0} * \log \left(1+\frac{1}{Y_{1}^{(2)}}\right)(x), \\
& \log Y_{2}^{(1)}(x)=G_{0} * \log \left[\frac{\left(1+Y_{3}^{(1)}\right)(x)\left(1+Y_{1}^{(1)}\right)(x)}{\left(1+\left(Y_{1}^{(2)}(x)\right)^{-1}\right)}\right] \\
& \left.\log Y_{m}^{(1)}(x)=G_{0} * \log \left(1+Y_{m-1}^{(1)}\right)(x)+G_{0} *\left(1+Y_{m+1}^{(1)}\right)(x)\right), \quad \text { for } m \geq 3, \\
& \log Y_{1}^{(a)}(x)=G_{0} * \log Y_{1}^{(a-1)}(x)+G_{0} * \log Y_{1}^{(a+1)}(x), \quad \text { for } a \geq 3, \\
& \log Y_{2}^{(2)}(x)=e_{3}(u, x)+G_{0} * \log \left(1+\left(Y_{1}^{(2)}(x)\right)^{-1}\right)-G_{0} * \log \left(1+Y_{2}^{(1)}\right)(x),
\end{aligned}
$$




$$
\begin{aligned}
\log Y_{1}^{(2)}(x) & =G_{0} * \log \left[\frac{\left(1+Y_{2}^{(2)}(x)\right)}{\left(1+\left(Y_{1}^{(3)}(x)\right)^{-1}\right)\left(1+\left(Y_{1}^{(1)}(x)\right)^{-1}\right)}\right] \\
& =e_{2}(u, x)+G_{0} * \log \left[\frac{\left(1+\left(Y_{2}^{(2)}(x)\right)^{-1}\right)}{\left(1+\left(Y_{1}^{(3)}(x)\right)^{-1}\right)\left(1+Y_{1}^{(1)}(x)\right)}\right] \\
e_{3}(u, x) & =N \int \frac{e^{-|k|-i k x} \sinh k u}{2 k \cosh k / 2} \mathrm{dk}
\end{aligned}
$$

$\Lambda_{1}^{(1)}(u, x)$ in terms of $Y_{m}^{(a)}$ reads,

$$
\begin{aligned}
\Lambda_{1}^{(1)}(u, x) & =\log \phi_{+}(x+i) \phi_{-}(x-i)+\sum_{j=1}^{\infty} K_{j} * \log \left(1+\left(Y_{j}^{(1)}\right)^{-1}\right), \\
K_{j}(x) & =\frac{j}{x^{2}+(j / 2)^{2}} .
\end{aligned}
$$

Now let $N \rightarrow \infty$ under identifications,

$$
\begin{aligned}
\alpha_{n} & =Y_{n}^{(1)}, \quad(n \geq 1) ; \quad\left(\alpha_{0}=1\right) \\
\beta_{1} & =Y_{1}^{(2)}, \quad \beta_{2}=Y_{2}^{(2)}, \\
\gamma_{s} & =1 / Y_{1}^{(s+2)} .
\end{aligned}
$$

Then the resultant TBA equations are nothing but the ones in [41], after taking some appropriate linear combinations

$$
\begin{aligned}
\log \alpha_{n} & =+G_{0} * \log \left[\left(1+\alpha_{n+1}\right)\left(1+\alpha_{n-1}\right)\right] \\
& -\delta_{n, 1}\left(2 \pi G_{0} / T+\log \left(1+1 / \beta_{1}\right)-\delta_{n, 2} \log \left(1+1 / \beta_{2}\right), \quad n \geq 1,\right. \\
\log \gamma_{s} & =+G_{0} * \log \left[\left(1+\gamma_{s+1}\right)\left(1+\gamma_{s-1}\right)\right]+\delta_{s, 1} G_{0} * \log \left(1+1 / \beta_{1}\right), \quad s \geq 1, \\
\log \beta_{1} & =-2 \pi G_{0} / T+G_{0} * \log \left[\frac{\left(1+\beta_{1}\right)}{\left(1+\alpha_{1}\right)\left(1+\gamma_{1}\right)}\right], \\
\log \beta_{2} & =-2 \pi G_{1} / T+G_{0} * \log \left[\frac{\left(1+1 / \beta_{1}\right)}{\left(1+\alpha_{2}\right)}\right], \\
f & =1-\sum_{n} K_{n} * \log \left(1+1 / \alpha_{n}\right) .
\end{aligned}
$$

Note that the present choice of Hamiltonian corresponds to $\mu+U / 2=0$. 


\section{Excited state TBA}

So far we have shown that the quantum transfer matrix formalism for the largest eigenvalue of $\Lambda_{1}^{(1)}$ with adequate information on analyticity properties directly relates the functional relations with the TBA equations. The investigation of other eigenvalues naturally leads to the excited state TBA equations, which characterize correlation lengths at finite temperatures. Note that the functional relations are valid irrespective of the sectors under consideration. The only difference to the "ground state" is the modification of the analyticity conditions by additional zeros or singularities entering into the strips where auxiliary functions in the ground state are strictly analytic and non-zero [57,61]. Let us examine explicitly the spin excitation for the solvable $t-J$ model.

For the ground state, we have $\frac{N}{2} x_{j}^{(1)}$ and $\frac{N}{2} x_{j}^{(2)}$ Bethe ansatz roots complex conjugate to each other. This time, we also have such complex conjugate pairs of BAE solutions, however, the number of solution is $N / 2-1$ for each. This does not seem to be a serious modification of the ground state, however it results into considerable differences in the zeros for $\Lambda_{m}^{(a)}$. We find $N-2$ zeros for $\Lambda_{m}^{(1)}$ on slightly curved lines close to $\Im x \sim \pm(m+1) / 2$ as for the ground state. Note that two zeros are missing on these lines. Instead we find (by numerical investigations for vanishing chemical potential) the additional structure:

(1) $\Lambda_{m}^{(1)}$ has zeros at $x_{m, \pm}^{(1)}$ on the real axis. The strictly largest eigenvalue is given by two symmetrically distributed zeros $x_{m, \pm}^{(1)}= \pm x_{m}^{(1)}$.

(2) $\Lambda_{1}^{(a)}, a \geq 2$, has zeros at $\pm\left(x_{1}^{(2)}+(a-2) i / 2\right)$ on the imaginary axis. We find $x_{1}^{(2)}$ is pure imaginary and $\left|x_{1}^{(2)}\right|>1 / 2$.

As a corollary from the identity $\widetilde{\Lambda_{m}^{(2)}}=\widetilde{\Lambda_{1}^{(m+1)}}$ we get

(3) $\Lambda_{m}^{(2)}$ has zeros at $\pm\left(x_{1}^{(2)}+(a-1) i / 2\right)$ on the imaginary axis.

Note that the behavior of zeros (2) is consistent with the "trivial" T-system,

$$
\widetilde{\Lambda_{1}^{(a)}}(x-i / 2) \widetilde{\Lambda_{1}^{(a)}}(x+i / 2)=\widetilde{\Lambda_{1}^{(a+1)}}(x) \widetilde{\Lambda_{1}^{(a-1)}}(x), a=3, \cdots, \infty .
$$

Thus, in terms of the $Y$ functions, we find the following zeros and singularities in the strip $\Im x \in\{-1 / 2,1 / 2\}$,

$$
\begin{aligned}
& Y_{m}^{(1)}(x)=0, \quad \text { at } x=x_{m-1, \pm}^{(1)}, x_{m+1, \pm}^{(1)}, \\
& Y_{1}^{(2)}(x)=\infty, \quad \text { at } x=x_{1, \pm}^{(1)} .
\end{aligned}
$$

In order to have ANZC functions in the strip we must modify the $\widetilde{Y_{m}^{(a)}}$ functions of section 4 further to

$$
\begin{aligned}
& \widetilde{Y_{m}^{(1)}} \rightarrow \widetilde{Y_{m}^{(1)}} \prod_{\sigma= \pm} \tanh \frac{\pi}{2}\left(x-x_{m+1, \sigma}^{(1)}\right) \tanh \frac{\pi}{2}\left(x-x_{m-1, \sigma}^{(1)}\right), \\
& \widetilde{Y_{1}^{(2)}} \rightarrow \widetilde{Y_{1}^{(2)}} / \prod_{\sigma= \pm} \tanh \frac{\pi}{2}\left(x-x_{1, \sigma}^{(1)}\right) .
\end{aligned}
$$


The resultant $\widetilde{Y_{m}^{(a)}}$ are now ANZC functions in the strip. Repeating the same argument as in section 4 , we thus arrive at

$$
\begin{aligned}
\log Y_{1}^{(1)}(u, x) & =e_{1}(u, x)+G_{0} * \log \frac{\left(1+Y_{2}^{(1)}\right)}{\left(1+1 / Y_{1}^{(2)}\right)}(x)+\sum_{\sigma= \pm} \log \left(\tanh \frac{\pi}{2}\left(x-x_{2, \sigma}^{(1)}\right)\right) \\
\log Y_{m}^{(1)}(u, x) & \left.=G_{0} * \log \left(1+Y_{m-1}^{(1)}\right)\left(1+Y_{m+1}^{(1)}\right)\right)(x) \\
& +\sum_{\sigma= \pm} \log \left(\tanh \frac{\pi}{2}\left(x-x_{m+1, \sigma}^{(1)}\right) \tanh \frac{\pi}{2}\left(x-x_{m-1, \sigma}^{(1)}\right)\right), \quad \text { for } m \geq 2 \\
\log Y_{1}^{(2)}(u, x) & =e_{2}(u, x)-G_{1} * \log \left(1+\frac{1}{Y_{1}^{(2)}}\right)(x)-G_{0} * \log \left(1+Y_{1}^{(1)}\right)(x) \\
& -\sum_{\sigma= \pm} \log \left(\tanh \frac{\pi}{2}\left(x-x_{1, \sigma}^{(1)}\right)\right)
\end{aligned}
$$

and the $x_{m, \sigma}^{(1)}$ satisfy,

$$
Y_{m}^{(1)}\left(u, x=x_{m, \sigma} \pm i / 2\right)=-1
$$

In the new integral equations the convolutions are still evaluated with integral contours along the real axis. In this way, we have derived quite naturally excited state TBA equations for spin excitations. We want to stress once more the meaning of these excitations. The corresponding eigenvalue determines via (2.7) the decay of static correlation functions, it is not related to dynamical correlations or "excited energies at finite temperature" [1]. For other types of excitations, one needs to identify the patterns and locations of zeros and singularities of the auxiliary functions. This is possible for small Trotter numbers, as we know explicitly the spectrum from "brute force" numerical calculations. We expect the so obtained patterns would be valid also in the limit $N \rightarrow \infty$. Lastly, we want to comment on our derivation of the TBA equations on the basis of ANZC properties. It appears that any solution to the integral equations is a solution of the functional equations and thereby yielding an eigenvalue to the quantum transfer matrix. The question remains whether the obtained eigenvalues are the largest ones. This however, is a byproduct of this section. Any time we modify the ANZC properties by allowing zeros to enter the physical strips the eigenvalue is lowered. This is the analytic justification of the previously employed analytic properties of the largest eigenvalue. 


\section{Summary and Discussion}

In this paper, we have shown the equivalence of the fusion relations among the quantum transfer matrices and the TBA equations under appropriate assumptions about analyticities of auxiliary functions. This has been demonstrated by adopting two examples of 1D highly correlated electron systems.

In our approach the derivation of the TBA equations is based essentially on ANZC properties of the auxiliary functions satisfying the functional relations. In some sense, the string hypothesis is now replaced by ANZC assumptions. We like, however, to point out the following. First of all, the analyticity properties are easier to control. We have checked numerically the ANZC properties for various configurations and for several Trotter numbers up to $N=32$. Second, in contrast to deviations from the string hypothesis for the finite system, we do not observe numerically any violation of the latter assumption for finite Trotter numbers.

Another advantage in our approach is the straightforward derivation of "excited state TBA". The additional chemical potential terms in TBA equations in the CFT limit of previous studies $[33,34,35,32]$ is now replaced by the concrete objects, the contribution by the additional zeros $x_{m}^{(1)}$ of the fusion transfer matrices.

For the actual calculation of physical quantities such as the specific heat or correlation lengths, we must deal with, in principle, an infinite number of unknown functions and parameters $x_{m}^{(1)}$. Let us sketch the situation in the case of the $t-J$ model. In order to solve numerically the TBA equations (4.7.a)- (4.7.e) we truncate the infinite set of integral equations (4.7.b) to a finite one by approximating $\log Y_{m}^{(1)}(x)$, for $m \gg 1$ by its asymptotic value. We thus introduce the quantity $\log \left(Y_{m}^{(1)}(0) / Y_{m}^{(1)}(\infty)\right)$ as an estimate for the systematic error. To evaluate this, we use the explicit solutions to BAE for finite Trotter numbers $N$ together with functional relations, thereby allowing the evaluation of these quantities with high numerical precision. In the limit $m \gg 1$ we find the following asymptotic behavior

$$
\log \left(Y_{m}^{(1)}(0) / Y_{m}^{(1)}(\infty)\right) \sim C / m^{2} .
$$

For the example $N=12, \beta=1 / T=3.5$ (and vanishing external fields), we have $C \approx 6$. Thus we need more than 2500 auxiliary functions $Y_{m}^{(1)}$ to obtain a numerical error less than $10^{-6}$. This already may be a sign of difficulties in obtaining good numerical accuracy for the traditional TBA analysis.

Fortunately, as we already remarked in the introduction, we have an alternative approach where only a finite number of auxiliary functions is necessary $[21,24,25,26,27]$. Although the connection to the traditional TBA is obscure, this formulation is more efficient in numerical investigations for physical quantities at finite temperatures. For the $t-J$ model for instance, we need only three auxiliary functions for a closed set of non-linear integral equations. This results in a numerical accuracy better than $10^{-6}[25,26]$ for the specific heat, compressibility and other thermodynamic quantities. Now the evaluation of correlation lengths is under investigation. We hope to report on explicit results based on this formulation in the near future. 


\section{Acknowledgments}

The authors acknowledge financial support by the Deutsche Forschungsgemeinschaft under grant No. Kl 645/3-1 and by the research program of the Sonderforschungsbereich 341, Köln-Aachen-Jülich.

They would like to thank Z. Tsuboi for discussions and collaboration at the early stage of the present work. Furthermore, J.S. gratefully acknowledges the hospitality of the Institut für Theoretische Physik der Universität zu Köln.

\section{References}

[1] C.N. Yang and C.P. Yang, J. Math. Phys. 10 (1969) 1115.

[2] M. Gaudin, Phys. Rev. Lett. 26 (1971) 1301.

[3] M. Takahashi, Prog. Theor. Phys. 46 (1971) 401.

[4] M.Takahashi and M. Suzuki, Prog. Theor. Phys. 48 (1972) 2187.

[5] A.A. Vladimirov, Non-String Two-Magnon Configurations in the Isotropic Heisenberg Magnet, Technical Report P17-84-409, Joint Institute for Nuclear Research Dubna, (1984).

[6] A. Klümper and J. Zittartz, Z. Phys. B 75 (1989) 371.

[7] H.L. Eßler, V.E. Korepin and K. Schoutens, J. Phys. A25 (1992) 4115.

[8] G. Jüttner and B.D. Dörfel, J. Phys. A 26 (1993) 3105.

[9] A. Klümper and M.T. Batchelor, J. Phys. A 23 (1990) L189.

[10] A. Klümper, M.T. Batchelor and P.A. Pearce, J. Phys. A 24 (1991) 3111.

[11] F.C. Alcaraz and M.J. Martins, J. Phys. A 21 (1988) L381, ibid. 4397.

[12] Al.B. Zamolodchikov, Phys. Lett. B253, (1991) 391.

[13] H.M. Babujian and A.M. Tsvelick, Nucl. Phys. B265 (1986) 24.

[14] M. Suzuki, Phys. Rev. B 31 (1985) 2957.

[15] M. Suzuki and M. Inoue, Prog. Theor. Phys. 78 (1987) 787.

[16] T. Koma, Prog. Theor. Phys. 78 (1987) 1213.

[17] J. Suzuki, Y. Akutsu and M. Wadati, J. Phys. Soc. Japan 59 (1990) 2667.

[18] J. Suzuki, T. Nagao and M. Wadati, Int. J. Mod. Phys. B6 (1992) 1119-1180.

[19] M. Takahashi, Phys. Rev. B 43 (1991) 5788.

[20] A. Klümper, Ann. Physik 1 (1992) 540.

[21] A. Klümper, Z. Phys. B 91 (1993) 507.

[22] C. Destri and H.J. de Vega, Phys. Rev. Lett. 69 (1992) 2313.

[23] H. Mizuta, T. Nagao and M. Wadati, J. Phys. Soc. Japan 63 (1994) 3951.

[24] A. Klümper, T. Wehner and J. Zittarz, J. Phys. A. 30 (1997) 1897.

[25] G. Jüttner and A. Klümper, Euro. Phys. Lett. 37(1997) 335.

[26] G. Jüttner, A. Klümper and J. Suzuki, Nucl. Phys. B487 (1997) 650.

[27] G. Jüttner, A. Klümper and J. Suzuki, J. Phys. A. 30 (1997) 1881.

[28] I. Cherednik, in Proc. of the XVII International Conference on Differential Geometric Methods in Theoretical Physics, Chester, ed. A.I. Solomon, (World Scientific, Singapore, 1989)

[29] V. Chari and A. Pressley, L'Enseignement Math. 36 (1990) 267.

[30] V. Chari and A. Pressley, Comm. Math. Phys. 142 (1991) 261.

[31] A. Kuniba, T. Nakanishi and J. Suzuki, Int.J.Mod.Phys A9 (1994) 5215, ibid 5267. 
[32] A. Kuniba and T. Nakanishi, Mod. Phys. Lett. A7 (1992) 3487.

[33] M.J. Martins, Phys. Rev. Lett. 67 (1991) 419.

[34] P. Fendley, Nucl. Phys. B374 (1992) 667.

[35] T.R. Klassen, E. Meltzer, Nucl. Phys. B370 (1992) 511.

[36] A. Kuniba, T. Nakanishi and J. Suzuki, Mod. Phys. Lett. A8 (1993) 1649-1659.

[37] P. Dorey and R. Tateo, Nucl. Phys. B482 (1997) 639.

[38] V.V. Bazhanov, S.L. Lukyanov and A.B. Zamolodchikov, Nucl. Phys. B489 (1997) 487.

[39] Z. Tsuboi, preprint(1997) UT-KOMABA 97-8.

[40] P. Schlottmann, Phys. Rev. B 36 (1987) 5177.

[41] F.H.L. Eßler and V.E. Korepin, condmat/9307019.

[42] F.H.L. Eßler, V.E. Korepin and K. Schoutens, Phys. Rev. Lett. 68 (1992) 2960.

[43] B. Sutherland, Phys. Rev. B 12 (1975) 3795.

[44] P.A. Bares, G. Blatter, Phys. Rev. Lett. 64 (1990) 2567.

[45] P.A. Bares, G. Blatter and M. Ogata, Phys. Rev. B 44 (1991) 130.

[46] C.N. Yang Phys. Rev. Lett. 63 (1989) 2144.

[47] J. de Boer, V.E. Korepin, A. Schadschneider: Phys. Rev. Lett. 74, 789 (1995);

A. Schadschneider: Phase Transitions 5737 (1996).

[48] J.H.H. Perk and C. Schulz, Phys. Lett. A 84 (1981) 407.

[49] C.L. Schulz, Physica A 122 (1983) 71.

[50] J. Suzuki, J. Phys. A 25 (1992) 1769.

[51] R.Z. Bariev, J. Phys. A 27 (1994) 3381.

[52] A. Klümper, Int. J. Mod. Phys. B 11141 (1997)

[53] J. Suzuki, Phys. Lett. A195 (1994) 190.

[54] A. Kuniba and J. Suzuki, Comm. Math. Phys. 173 (1995) 225-264.

[55] A. Kuniba, Y. Ohta and J. Suzuki, (1995) J.Phys A 28 6211-6226.

[56] V.V. Bazhanov and N.Yu Reshetikhin, Int.J.Mod.Phys A4 (1989) 115.

[57] A. Klümper and P.A. Pearce, Physica A183 (1992) 304.

[58] For the representation theoretical meaning of this relation, see, T. Deguchi and P.P. Martin, Int. J. Mod. Phys. A7 (1992) 1A 165 and P.P. Martin and V. Rittenberg, Int. J. Mod. Phys. A7 (1992) 1B 707.

[59] Y.-K. Zhou and M.T. Batchelor, Nucl. Phys. B 490 [FS] (1997) 576.

[60] F. Ravanini, R. Tateo and A. Valleriani, Phys. Lett. B293 (1992) 361.

[61] For similar calculations on finite size correction for row-to-row transfer matrix of the Hard Hexagon Model, see A. Klümper and P.A. Pearce, Phys. Rev. Lett. 66 (1991) 974, J. Stat. Phys. 64 (1991) 13. 
This figure "fig1-1.png" is available in "png" format from: http://arxiv.org/ps/hep-th/9707074v1 
This figure "fig1-2.png" is available in "png" format from: http://arxiv.org/ps/hep-th/9707074v1 